ECCOMAS

Proceedia
EUROGEN 2021

$14^{\text {th }}$ ECCOMAS Thematic Conference on Evolutionary and Deterministic Methods for Design, Optimization and Control N. Gauger, K. Giannakoglou, M. Papadrakakis, J. Periaux (eds.) Streamed from Athens, Greece, 28-30 June 2021

\title{
BRIDGES MONITORING: AN APPLICATION OF AI WITH GAUSSIAN PROCESSES
}

\author{
Rebecca Asso ${ }^{1}$, Raffaele Cucuzza ${ }^{2}$, Marco M. Rosso ${ }^{2}$, Davide Masera ${ }^{1}$ and Giuseppe \\ C. Marano ${ }^{2}$ \\ ${ }^{1}$ Masera Engineering Group s.r.l. \\ Corso Re Umberto 8, Torino (IT) \\ rebecca.asso@masera-eg.com \\ davide.masera@masera-eg.com \\ 2 Politecnico di Torino, DISEG \\ Corso Duca degli Abruzzi 24, Torino (IT) \\ raffaele.cucuzza@polito.it \\ marco.rosso@polito.it \\ giuseppe.marano@polito.it
}

\begin{abstract}
Monitoring and assessment of existing infrastructures is gaining attention in nowadays society as aging degradation reduces the residual structural safety. This work aims at providing a competitive procedure for the monitoring of bearings of bridges, based on data analysis coming from the use of strain gauges. It proposes an unsupervised learning (USL) approach based on a single-valued performance indicator to monitor the global behavior of bearings as critical components in a viaduct. The procedure for data analysis is exploiting the well-known Gaussian Processes to analyze and correlate horizontal bearing displacements and environmental temperature, as primary source of deformation of the deck. With this approach, we combine measurements from twelve sensors to design a robust performance indicator that is only weakly affected by temperature variations. Data exploitation and manipulation is described, from data acquisition and selection to the interpretation of results. Meanwhile the availability of a huge amount of information coming from the monitoring of big structures is no more an issue, the management of such indications is not always clear and efficient. For this reason, the method proposed by this paper can be of support in dealing with real-time monitoring of bridge bearings, maximizing unexpected maintenance and increasing the safety of the bridge.
\end{abstract}

Keywords: bridge bearing; machine learning, Gaussian processes, Structural Health Monitoring, Big Data 


\section{INTRODUCTION}

Nowadays, monitoring and assessment of structures are one of the most interesting and urgent topics that occupy a central role in research. Bridges are no exception: due to their location and structure, they are often subjected to critical environmental conditions and load cases. Over the last decades, technological support radically increased, shifting the research attention in the direction of data management, rather than data acquisition. Sensors have reached an excellent level regarding precision, resistance and maintenance, data is available remotely with an automatic upload on a cloud, in some cases it is also possible to acquire information in a wireless mode. The exploitation of advanced monitoring systems provides direct benefits in management and utilization of structures under service conditions, allowing an optimization of repairing actions and increase in safety. Structural Health Monitoring (SHM) tools can implement and interpret data coming from the monitoring system, providing threshold values, alarm conditions, trends and much more [1]. Depending on the type of sensor and monitoring architecture defined, local or global indications of the structure are provided. With the exploitation of distributed sensors such as distributed Optic Fibres, the overall behavior of the bridge is studied and reported with a certain variability and precision, while with local sensors the information acquired is usually with higher accuracy and sampling rate, but referred to a limited area only. The choice on which system to be adopted is driven by prior knowledge on the most critical elements, coming from visual inspections or based on Finite Elements Models. SHM aims at identifying and tracking those features that most witness the presence and development of damage. To this end, the monitored element is compared to a reference state referred as undamaged and the system is defined Model-Based or Data-Based [2] depending on the type of method used for the definition of the undamaged scenario.

Model-based SHM systems are traditionally used in structural engineering, since they require the exploitation of models (usually Finite Element Models) that represent the geometry, load cases, boundaries and known degradation processes. The model is required to be matching the real structure monitored, therefore some chosen characteristics are varied until the response is in line with the one of the monitored structure. The problem of identification of the most sensitive features is solved with the FE analysis, that provides threshold values too.

Data-driven SHM systems are only studying and manipulating monitoring data, developing algorithms that after a training phase are able to work autonomously. Since they are only relying on observed features, they are free from assumptions and simplifications that could affect the FE models, they provide strong tools for a long monitoring plan, defining those functions that describe the behavior and response of the structure under unknown loads. However, this approach has some limitations when a fast-monitoring activity is necessary, since algorithms require an initial calibration period, based on data coming from the structure. This period is not effective for the monitoring purposes, it is only providing information about the structural response, forming the comparison data-set for the following monitoring stages. Calibration of the algorithms can be supervised or unsupervised: if information about both damaged and undamaged stages are known, the learning method is defined as supervised, while if the health condition is not known the learning approach is called unsupervised. Most civil engineering structural monitoring is in unsupervised mode, in fact the correct and incorrect behavior of the structure is not given, the possible and current damage scenarios are unknown, and data registered is hardly linked to a defined health condition. There are a few known case studies where the damage was gradually introduced, allowing a supervised mode calibration [3]. After the algorithms have fulfilled the training phase, they are applied to a new data-set of test data, that are classified as belonging to a damaged or undamaged structure. This second phase is called test phase and represents the moment of active monitoring. 
In this paper, the data-based approach proposed is applied to the mobile bearings of an Orthotropic Steel Deck (OSD), and it is described in all the steps that go from data cleansing to threshold definition and data management. The proper transfer of loads from the superstructure to the substructure is affected by the correct behavior of bearings, that in design phase are defined as mobile or fixed to avoid the contrast caused by environmental actions (temperature variations) and at the same time provide stability to the structure. If a mobile bearing develops damage at the sliding or rolling surface, it does not allow the demanded movements of the superstructure: it causes the development of a friction (or increase in stiffness), and the introduction of critical stresses at local scale, decreasing safety and durability of the structure. Proper maintenance of the structure must be considering the health state of the bearings. The proposed method offers a SHM tool for early warning on the real condition of bridges, which can assist managers in scheduling the maintenance actions at network level. The methodology also helps to complement visual inspections for individual elements with more quantitative insight regarding the global behavior of the structure. We envision the present work as a complementary tool that should work together with other SHM assessment practices, including deterministic approaches to locate and quantify the damage.

The paper is organized as follows: in the next chapter there is a brief introduction regarding the state-of-the-art in bearings monitoring and gaussian processes, then in chapter 3 a first insight in the FE model development is shown. Chapter four describes the procedure followed for data preprocessing and postprocessing and chapter five presents and discusses the obtained results.

\section{STATE OF THE ART}

Continuous monitoring of structures is gaining importance in nowadays society, deeply affecting focus and interest in research fields. In recent years, many catastrophic events have shown the importance of a continuous assessment of the response of structures in service, as a tool for defining the state of health and damage conditions.

\subsection{Bearings of bridges monitoring}

Each structural part is influencing the correct behavior of the structure, and bearings are crucial elements since they allow the correct flow of stresses and strains from the superstructure to the substructure. Their direct correlation with temperature variations can be appreciated with strain gauges and temperature sensors. An application of the AI in bearings monitoring has been observed in [4]-[8], where the correlation between environmental temperatures and the bridge movements could witness a state of health of the bearing. In the case of older steel bearings, it is possible to face corroded and critically damaged elements (Figure 1 ), and in some other cases the original design and construction allow no direct inspections to verify the health of supports.

To better organize repairing and replacements, it is first necessary to know what is the conditions of damage of the element, in direct or indirect way. In most cases, it is possible to easily access the location of the bearing, with a by bridge or directly from an inspection path, but sometimes it is not possible to verify the condition of the bearings, as displayed in Figure 1. 


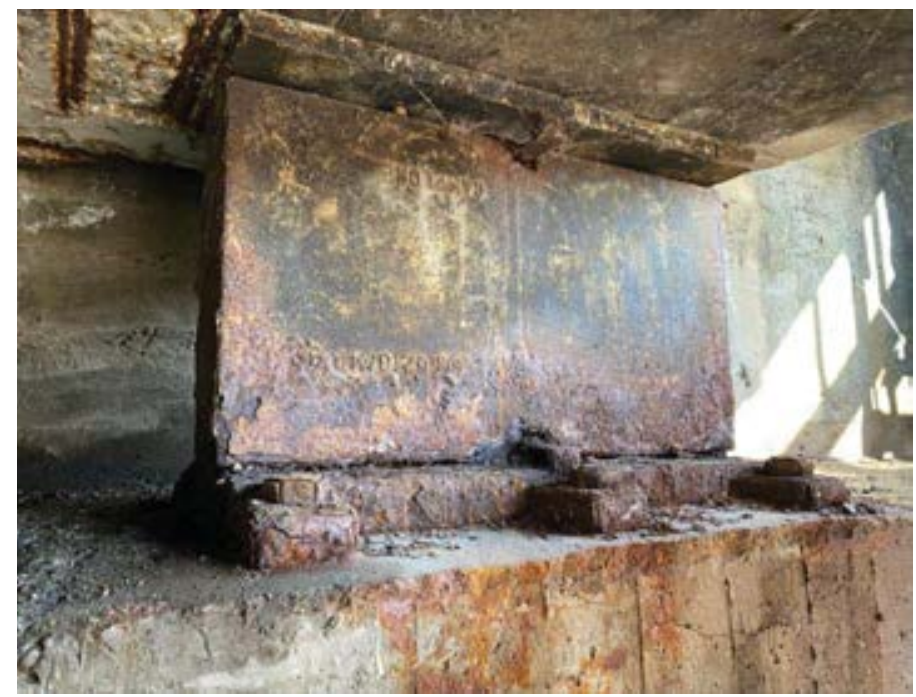

Figure 1: Environmental effects on steel bearings.

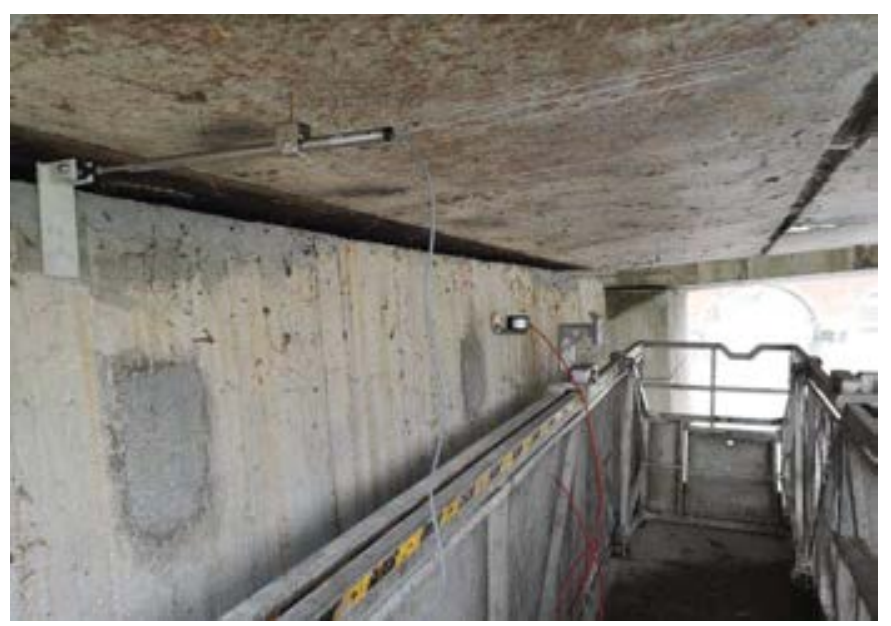

Figure 2: Application of sensors for monitoring "hidden" bearings

The benefits of a monitoring period of few months are several:

- One year of data collected from a structure in service forms a useful "bank" concerning the structural response, that can be a key point for future necessities. Most of the traditional monitoring activities have a black period of a few months where the monitoring setup needs to be calibrated, and this would be avoided.

- Sensors that are placed on the structure are highly performing for a long time and under critical environmental conditions, so they could be left in situ waiting for the following data sampling. Setup and sensors placing time and costs would be avoided.

- Maintenance of critical infrastructures is usually expensive and time-consuming, the proper schedule of repairing allows cost and time optimization for a higher structural safety.

- Data-driven SHM is overcoming the traditional problems and limits that are affecting model-based SHM, with low impacts on the operative life of the structure.

Moreover, the application of Artificial Intelligence (AI) to structural monitoring allows to develop algorithms that are able to define if the structure is damaged or not. This issue must 
not be underestimated since big structures like bridges and viaducts are characterized by properties that do not allow a direct identification of damage:

- Structural response is affected by environmental loads (thermic and wind), operational actions (traffic loads) and material behavior (creep and shrinkage).

- Each case study is unique, because of the loading history during its service life, structural materials, construction phases.

- Damage mechanisms are slow and take years to develop critical conditions.

- Variables that are monitored are affected by bias.

The innovation proposed in this paper is a method that is requiring a limited amount of data to be applicable and interpretable. Obviously, with the increase of information registered it is possible to have higher accuracy, but when a quick indication of the monitoring results is necessary, it is possible to safely apply this method.

\subsection{Gaussian Processes}

Gaussian Processes are a family of algorithms that can be extremely powerful to the end of regression and prediction. They represent stochastic processes that are described by their mean function $m(x)$ and covariance function $k\left(x, x^{\prime}\right)$ Eqs. (1)-(2), usually applied for the definition of a priori distribution in Bayesian inference [9]. In fact, a stochastic process is defined as the probability distribution of a sample of variables $Y\left(x^{(1)}\right), \ldots, Y\left(x^{(n)}\right)$ coherent to the input data given as a group of random variables $\{Y(x) \mid x \in X\}$.

$$
\begin{gathered}
m(x)=E[f(x)] \\
k\left(x, x^{\prime}\right)=E\left[(f(x)-m(x)) \cdot\left(f\left(x^{\prime}\right)-m\left(x^{\prime}\right)\right)\right]
\end{gathered}
$$

The Gaussian Process takes the form of:

$$
f(x) \sim G P\left(m(x), k\left(x, x^{\prime}\right)\right)
$$

Or, in the extended way:

$$
\left[\begin{array}{c}
f\left(x_{1}\right) \\
\vdots \\
f\left(x_{n}\right)
\end{array}\right] \sim G P\left(\left[\begin{array}{c}
m\left(x_{1}\right) \\
\vdots \\
m\left(x_{n}\right)
\end{array}\right],\left[\left(\begin{array}{ccc}
k\left(x_{1}, x_{1}\right) & \cdots & k\left(x_{1}, x_{n}\right) \\
\vdots & \ddots & \vdots \\
k\left(x_{n}, x_{1}\right) & \cdots & k\left(x_{n}, x_{n}\right)
\end{array}\right)\right]\right)
$$

Although it is generally agreed to initially assume a mean function equal to zero, the covariance function can be chosen among many possible functions. Their main requirement is the formation of a matrix with non-negative elements for every set of points, and this is obtained with a calibration of hyperparameters on the original group of variables.

Real data are usually affected by a white noise in background, that can be assumed to have a Gaussian distribution with zero mean and a certain standard deviation $\sigma_{n}^{2}$. In this case, the dependent variable $y$ is function of both components, as reported below.

$$
\begin{aligned}
p(f(x) \mid \theta) & \sim G P\left(m(x), k\left(x, x^{\prime}\right)\right) \\
\epsilon & \sim N\left(0, \sigma_{n}^{2}\right) \\
y & =f(x)+\epsilon
\end{aligned}
$$


GP exploits a probabilistic approach to define a robust unsupervised method, but the correct identification of training data is fundamental to avoid problems of under-fitting and overfitting.

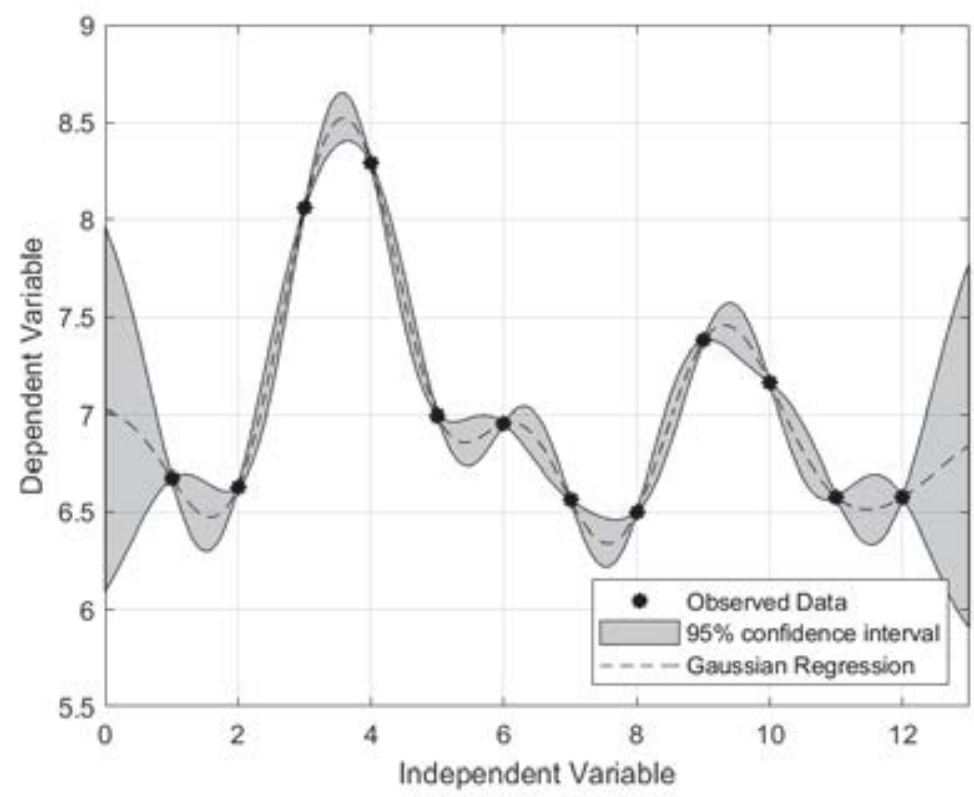

Figure 1: Gaussian Process

The application of Gaussian Processes allows to identify a regression function from known couples of variables, and a region where it is expected to have the future dependent variable once the independent is known, reported in Figure 1. The expectation probability is set on the basis of the covariance function.

\section{CASE STUDY: THE BRIDGE}

The case study consists of a continuous steel bridge composed by two independent carriageways, both supported by four reinforced concrete piers $35 \mathrm{~m}$ high (Figure 2 and Figure 3 ). The north carriageway is $307 \mathrm{~m}$ long $(93.5 \mathrm{~m}+120 \mathrm{~m}+93.5 \mathrm{~m})$ while the South is $279.5 \mathrm{~m}$ $(76.25 \mathrm{~m}+120 \mathrm{~m}+83.25 \mathrm{~m})$. The Orthotropic Steel Deck (OSD) is a box girder with variable height that is minimum at the two ends $(3 \mathrm{~m})$ and maximum and constant in the central span $(5 \mathrm{~m})$. The multidirectional supports consist in PTFE bearings that allow sliding in transverse and longitudinal directions, they are placed in all piers (X, XI, XII and XIII), while in the pier XI of both carriageways are placed the fixed supports. In piers X, XII and XIII are placed anti-seismic supports. 
LONCIUCWNAL WEW - NORIH CARRIACEMAY

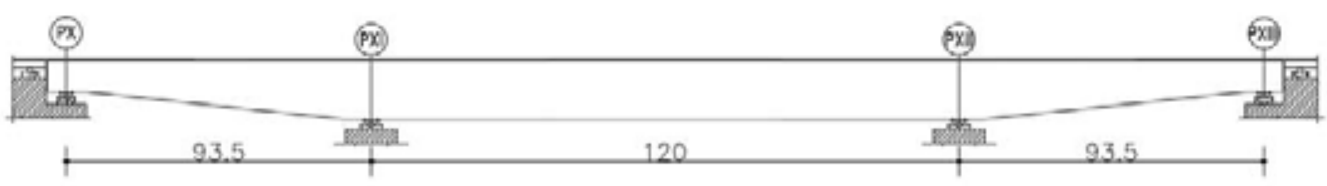

LONGITUWWL WEW - SOUTH CARRMGEWAY

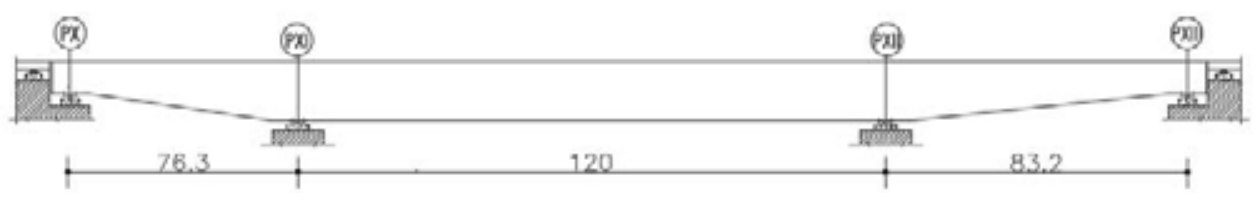

Figure 2: Longitudinal view of the two carriageways

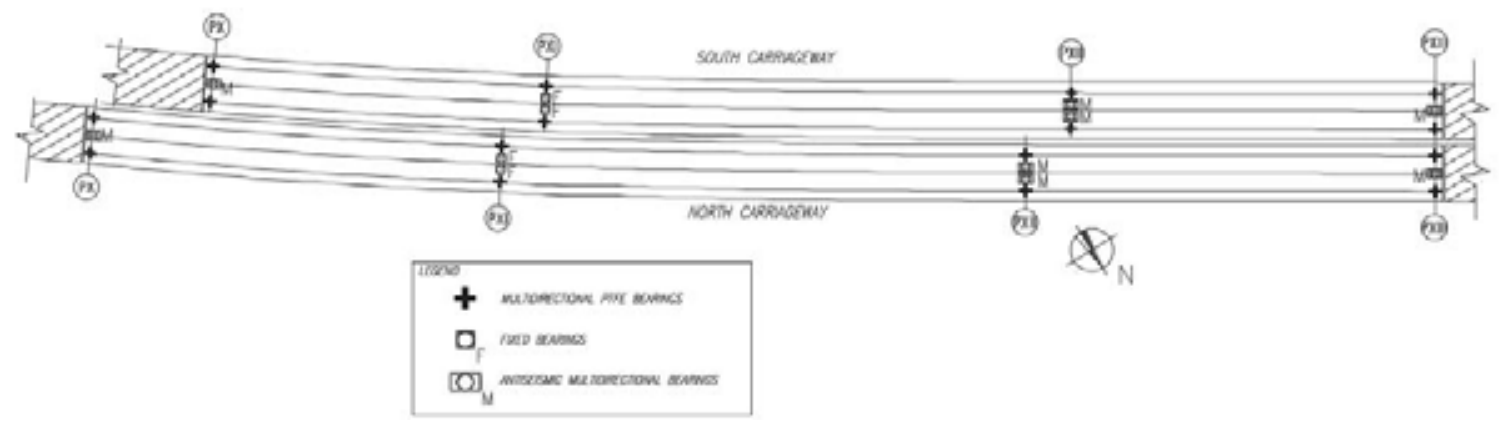

Figure 3: Top view of the two carriageways

All the mobile supports are monitored in the longitudinal direction with a total of 16 displacement sensors, and two temperature sensors are placed in the two ends of the bridge (pier $\mathrm{X}$ and pier XIII of the north carriageway). Data is communicated to acquisition boxes, that upload all the information gained to a cloud, accessible remotely. Since electricity is not provided to the structure, a solar panel has been necessary to reload the battery of each acquisition box, limiting the information sampling to one every 30 minutes to avoid battery issues.

\section{DATA ANALYSIS}

Data are downloaded from the cloud and analyzed. In Figure 4 it is shown the sensors positioning and nomenclature.

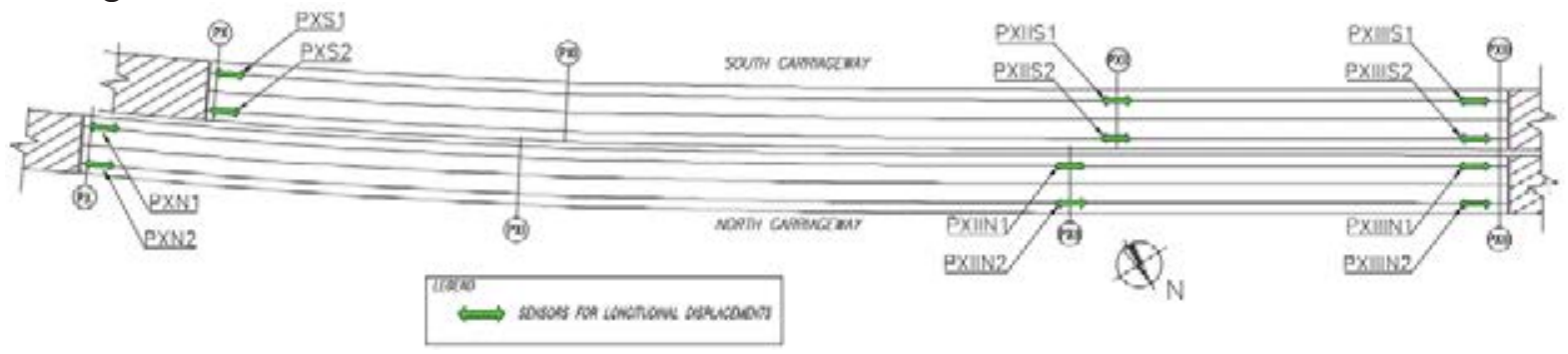

Figure 4: Sensors positioning and nomenclature 


\subsection{Preprocessing}

At first, data must be cleansed: it is not rare to have malfunctions of sensors, therefore it is necessary to check the incoming information to witness a proper sampling [10]. Outliers are detected exploiting a statistical approach: normal objects are belonging to regions with high probability, while outliers are in low-probability [11]. Afterwards, they must be rescaled to allow a direct comparison between displacement and temperature variations which are in two different unit systems [12].

$$
S_{i}=\frac{x_{i}-\mu}{\sigma}
$$

Finally, the correlation between displacements and temperatures is evaluated with the Pearson coefficient as shown in Eq. (7):

$$
P_{X Y}=\frac{\sigma_{X} \cdot \sigma_{Y}}{\sigma_{X Y}}
$$

- $+0.7<\rho_{X Y}<+1.0$ : high direct correlation between the two variables;

- $0.3<\rho_{X Y}<+0.7$ : medium direct correlation between the two variables

- $0.0<\rho_{X Y}<+0.3$ : small to zero direct correlation between the two variables;

- $-0.3<\rho_{X Y}<0.0$ : small to zero indirect correlation between the two variables;

- $-0.7<\rho_{X Y}<-0.3$ : medium indirect correlation between the two variables;

- $-1.0<\rho_{X Y}<-0.7$ : high indirect correlation between the two variables.

It is expected to have a strong correlation between the displacements and temperature variations, and this is confirmed from the evaluation of the Pearson coefficient as shown in Figure 5 .

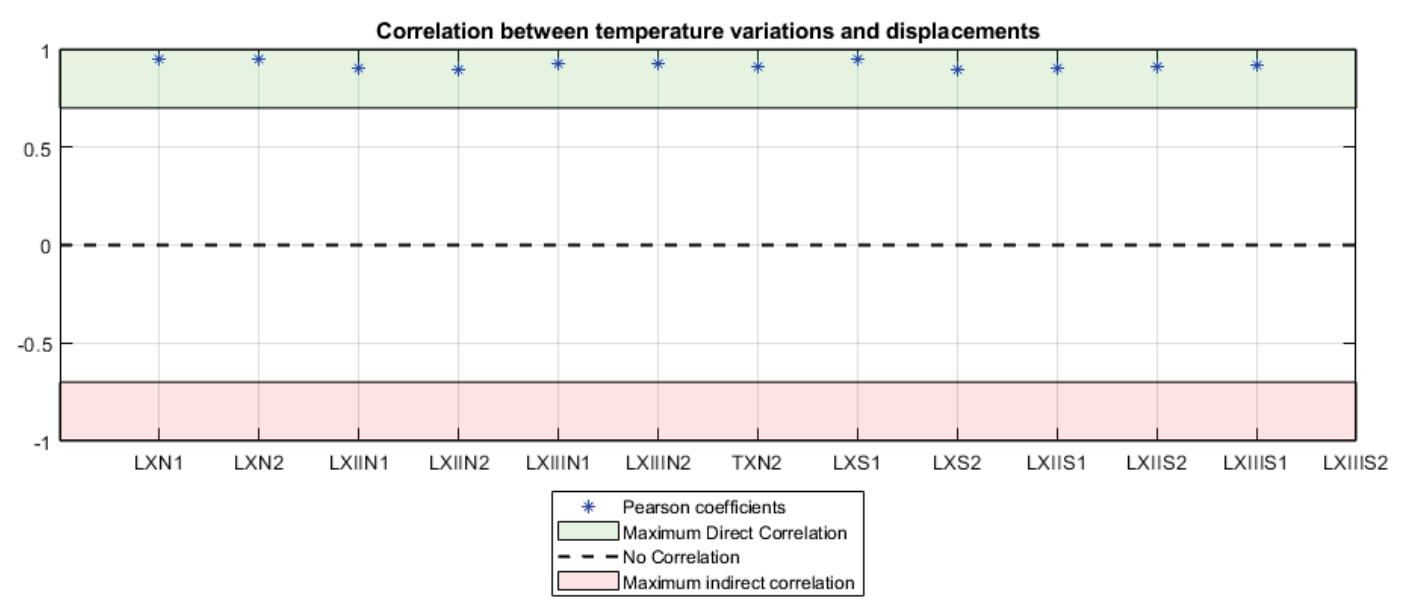

Figure 5: Pearson coefficients of sensors

\subsection{Postprocessing}

Data postprocessing is based on an equivalent analytical model of a beam supported by a hinge and a roller support, in Figure 6. This latter is not allowed to move freely but it is connected to a spring with a certain equivalent stiffness $K$, which is the property of each bearing 
to be evaluated and monitored. The beam is subjected to a uniform temperature variation that causes an axial force in the element, evaluated as $F$ in Eq. (8):

$$
F=\alpha \cdot \Delta T \cdot A \cdot E[k N]
$$

Where:

- $\alpha\left[{ }^{\circ} \mathrm{C}^{-1}\right]$ is the thermal coefficient of the material of the deck;

- $\Delta T\left[{ }^{\circ} \mathrm{C}\right]$ is the uniform temperature variation;

- $A\left[\mathrm{~mm}^{2}\right]$ is the cross-sectional area;

- $E\left[\mathrm{kN} / \mathrm{mm}^{2}\right]$ is the deck stiffness.

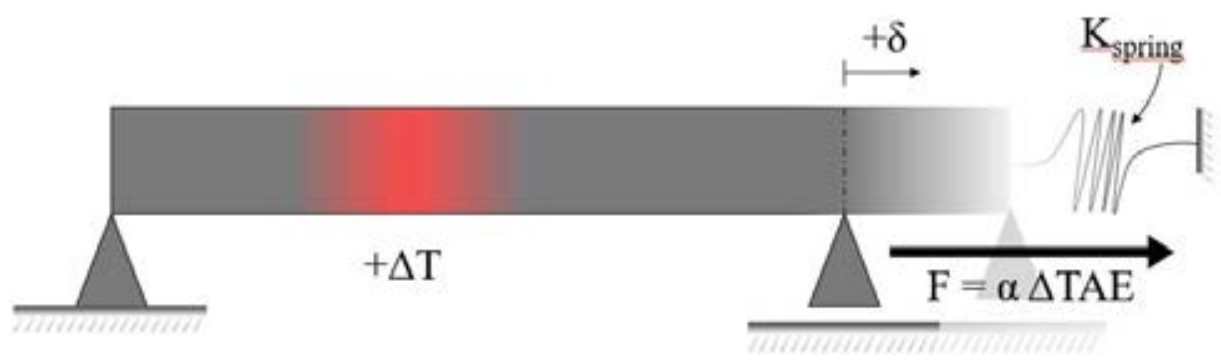

Figure 6: Static scheme

The gaussian distribution of displacements and temperature variations highlights the mean stiffness of the spring, the minimum and the maximum values that can maximize the interval containing $95 \%$ of the registered data. All information coming from the Gaussian processes are exploited at their best to maximize the monitoring activities at their early stage.

In fact, it is not always possible to wait until a proper amount of information is available for data analysis and decision making, and for this end this procedure has been developed: after a few months of data, it is possible to have a preliminary indication of the bearings' health.

Data is split in two groups: the first sample (S01) provides a term of comparison and identifies an indication of stiffness for each bearing, while the second sample (S02) is assessed to belong to the same population of data. The area which is defined by the $95 \%$ of S01 also defines the threshold values that must be valid for S02: since this group is characterized by a lower variability, it is also expected to decrease its dispersion in the Gaussian Process. The first 15 days belong to the sample S01, and all the data from the $16^{\text {th }}$ day belong to the $2^{\text {nd }}$ sample. It is expected to update the elements of S01 sample at the season change, due to the non-linear behavior of the bearings with temperature variations.

\section{RESULTS AND DISCUSSION}

The strong correlation between temperature variations and displacements of the bearings is first highlighted by Pearson coefficients, and then confirmed from the evaluation of the equivalent stiffness of the bearing. The mean stiffness is associated to the GP regression, while the maximum and the minimum stiffnesses are obtained from the maximization of the $95 \%$ confidence interval.

After one month of monitoring ( $\mathrm{S} 01=15$ days, $\mathrm{S} 02=15$ days), it is possible to identify the behavior of the bearings as shown in Figure 7. 


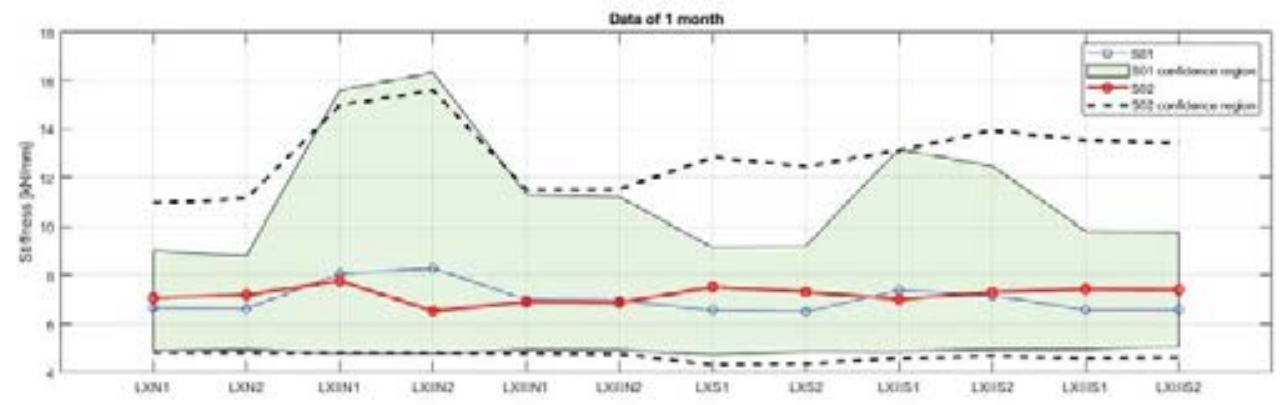

Figure 7: Data after one month of monitoring

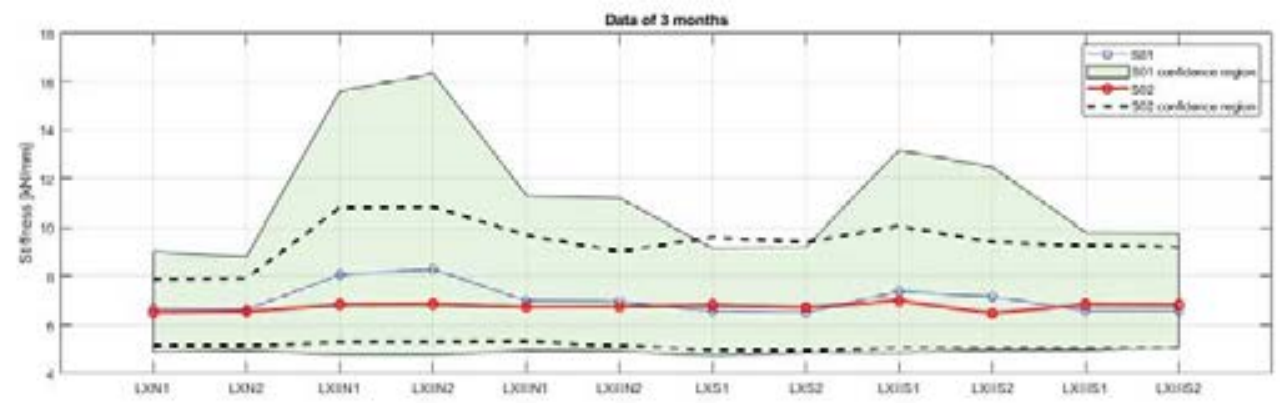

Figure 8: Data after two months of monitoring

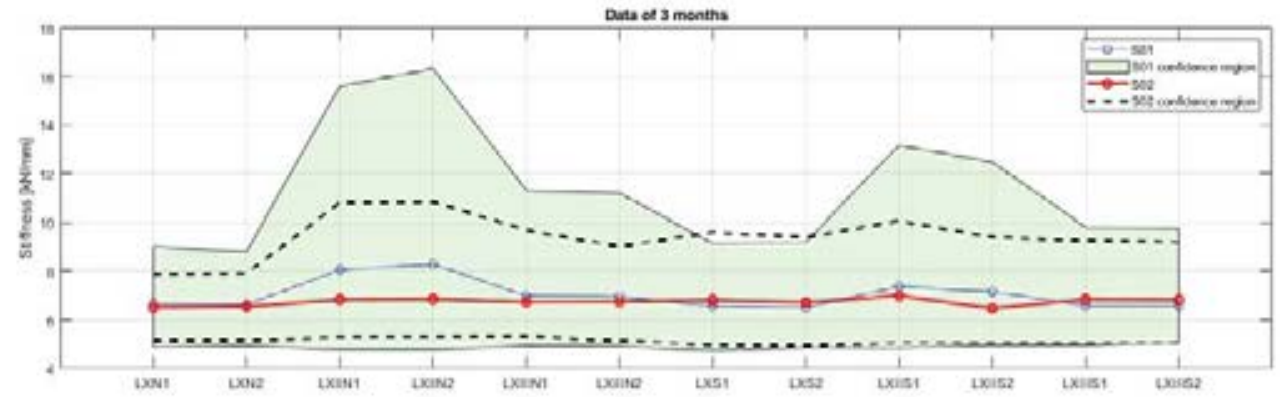

Figure 9: Data after three months of monitoring

As expected, data belonging to the S02 group are less dispersive with the increase of the monitoring duration (Figure 8 and Figure 9). The aim of the monitoring system is to assess that the bearing does not reach a level of stiffness too high, that is relative to a "hardening" behavior of the elements, meaning a possible change in the static scheme [13] of the bridge.

The evaluation of stiffnesses can be a good practical approach to visualize the bearings' health, but it could be affected by improper simplifications. To double check the correctness of data is possible to train a gaussian process on S01 and apply it on S02 data, obtaining a prediction of data. This suggested approach is computationally more expensive, but can better investigate anomalies, when detected.

\section{CONCLUSIONS}

The paper introduces a new procedure for the monitoring of bearings of bridges. It correlates displacements of the bridge deck and temperature variations, defining a fictitious stiffness of each bearing. This parameter is characterizing the health of the moving bearing: a small stiffness is representing an element that is free to move without causing excessive stresses in the piers. 
After a few months from the activation of the sensors it is possible to draw the following conclusions:

- A sampling frequency of two data each hour is enough to appreciate the effects of thermal loads on the structure.

- Incoming data must be verified to use correct information and check the health of the sensors.

- Gaussian Processes are valid tools to process this type of data, providing robust and engineering meaningful data for the monitoring aim.

- Variables that are influencing the stiffness K can be identified, such as dead loads, damage and age of the bearings, position of the element.

- This procedure can be support in management and interpretation of data coming from not fully known structures.

- This procedure can be applied to other parameters characterizing the structural response, such as rotations, curvatures, vertical displacements, crack width.

\section{APPENDIX}

The following graphs are showing the Gaussian Regressions with one, two and three months of data available.
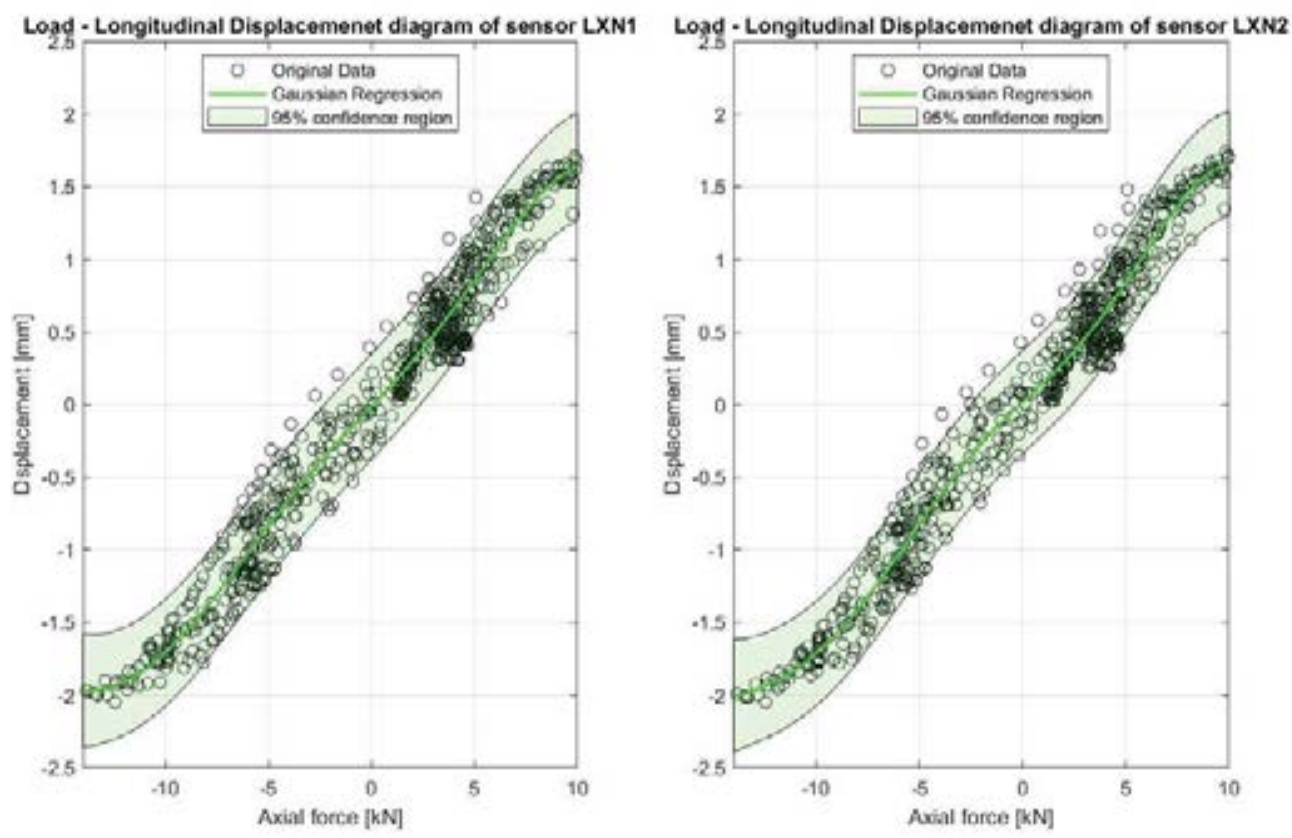

Figure 12: Gaussian Regression of LXN1 and LXN2 after one month of data 

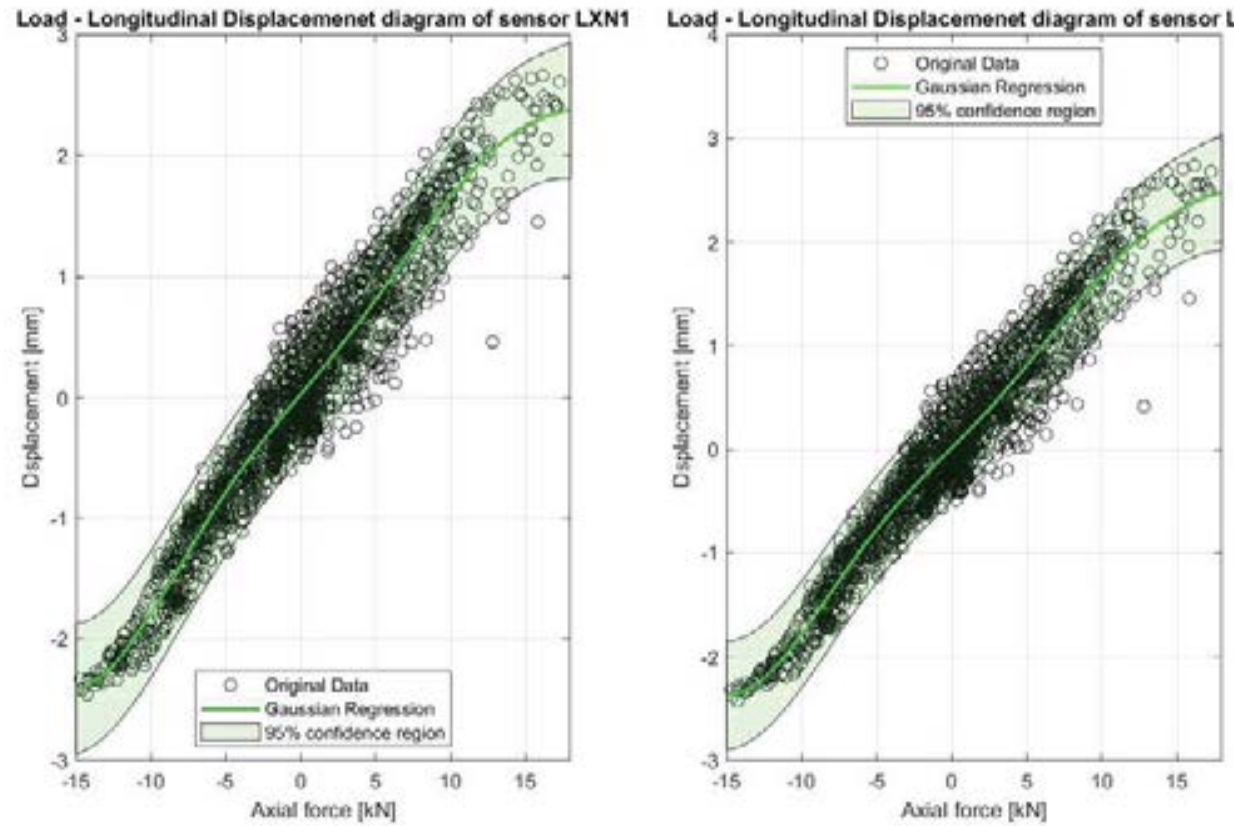

Figure 12: Gaussian Regression of LXN1 and LXN2 after two months of data
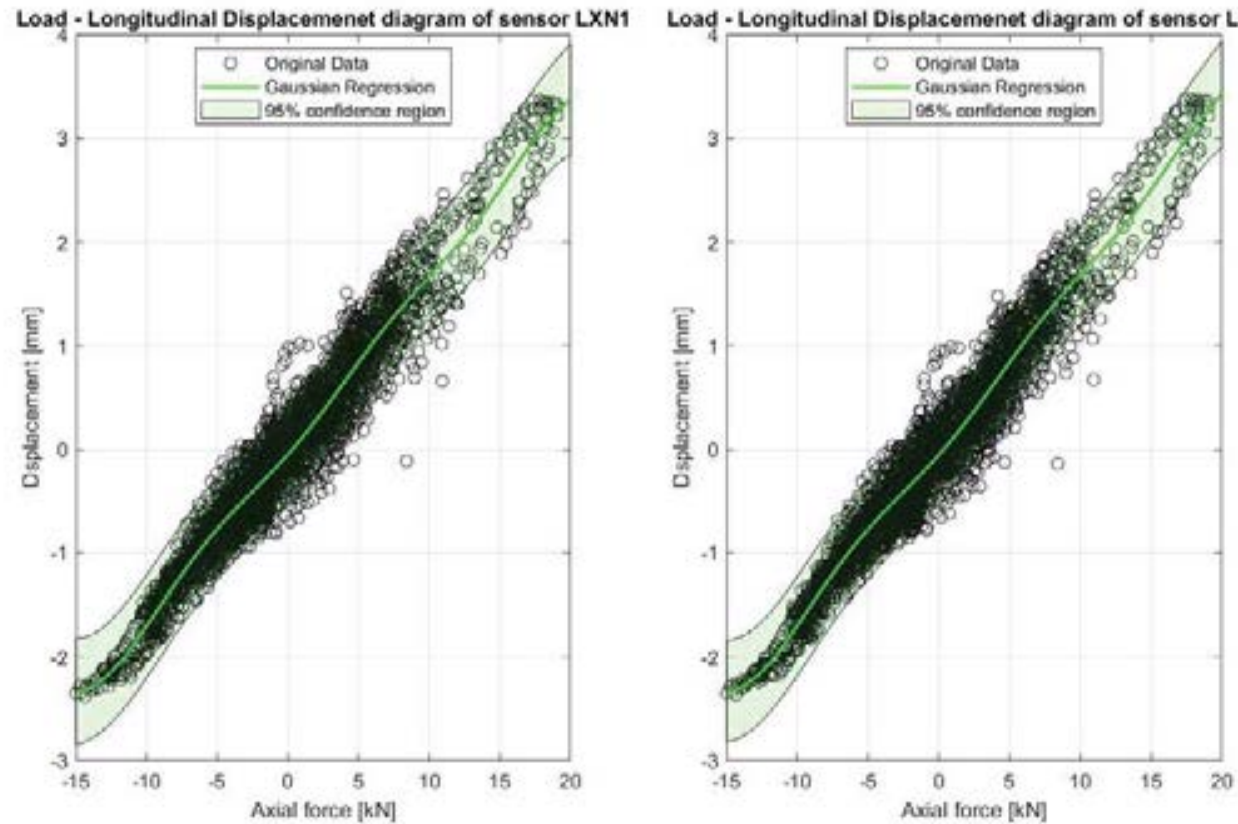

Figure 12: Gaussian Regression of LXN1 and LXN2 after three months of data 

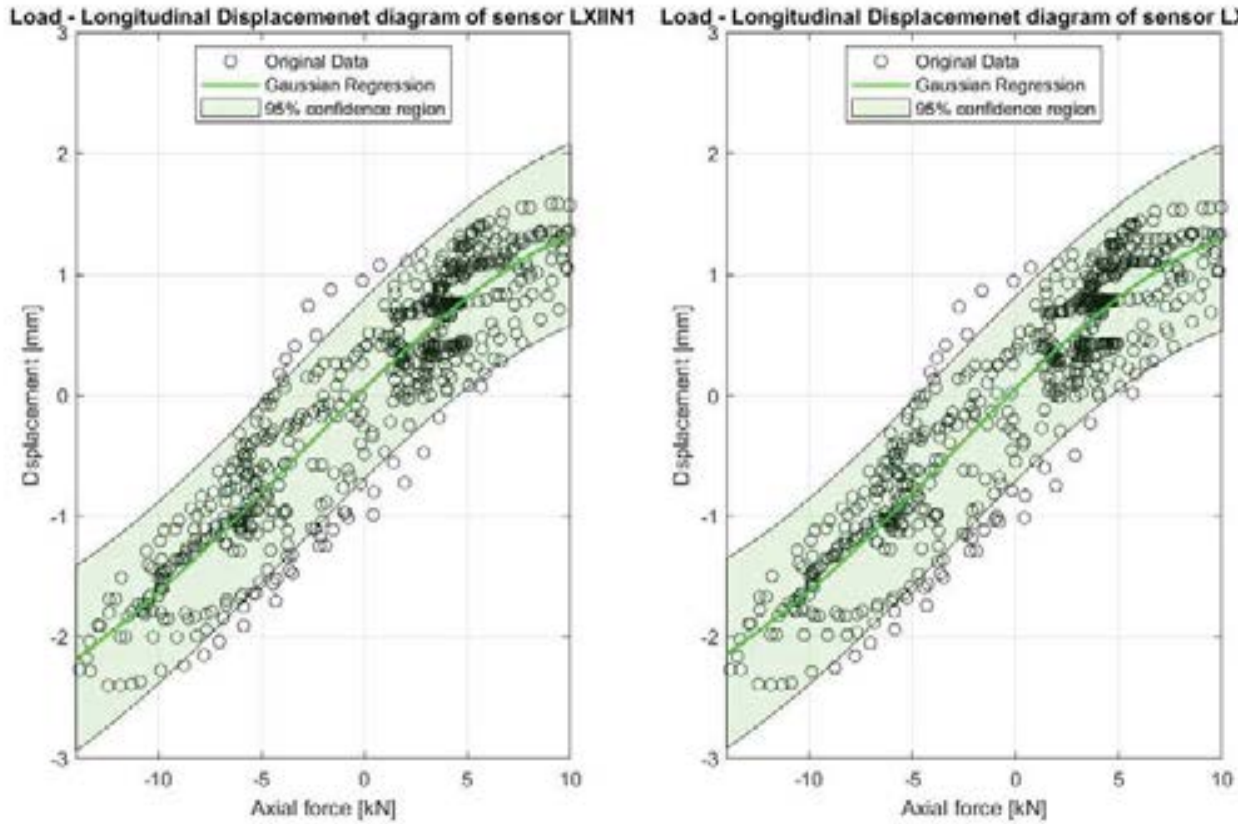

Figure 12: Gaussian Regression of LXIIN1 and LXIIN2 after one month of data
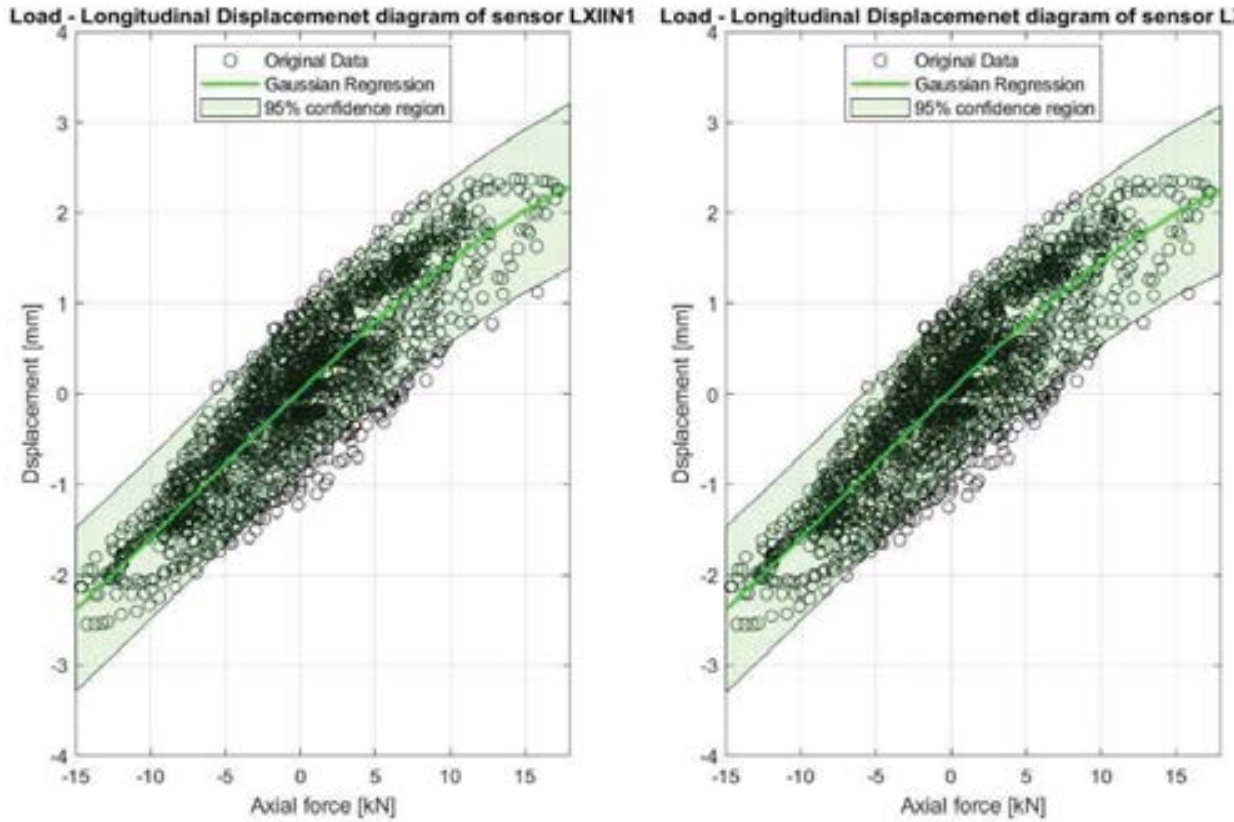

Figure 12: Gaussian Regression of LXIIN1 and LXIIN2 after two months of data 

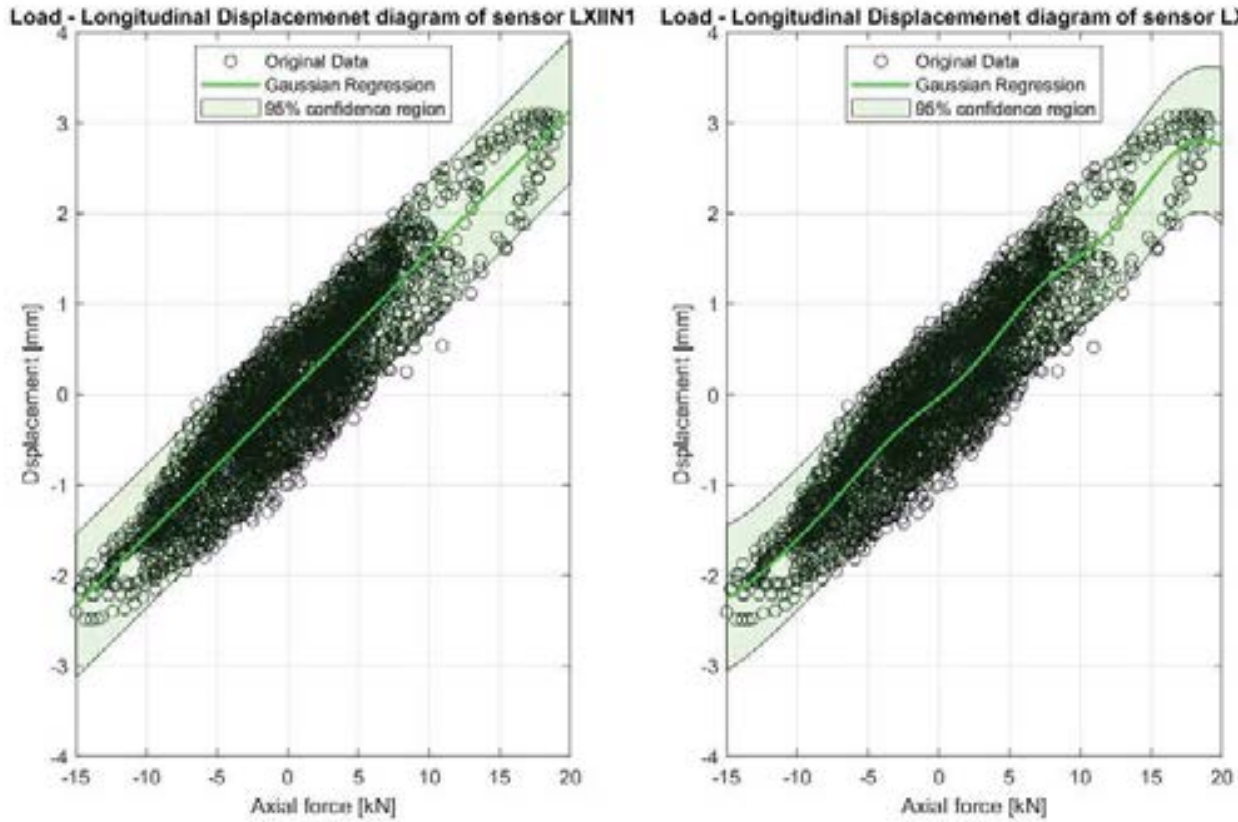

Figure 12: Gaussian Regression of LXIIN1 and LXIIN2 after three months of data
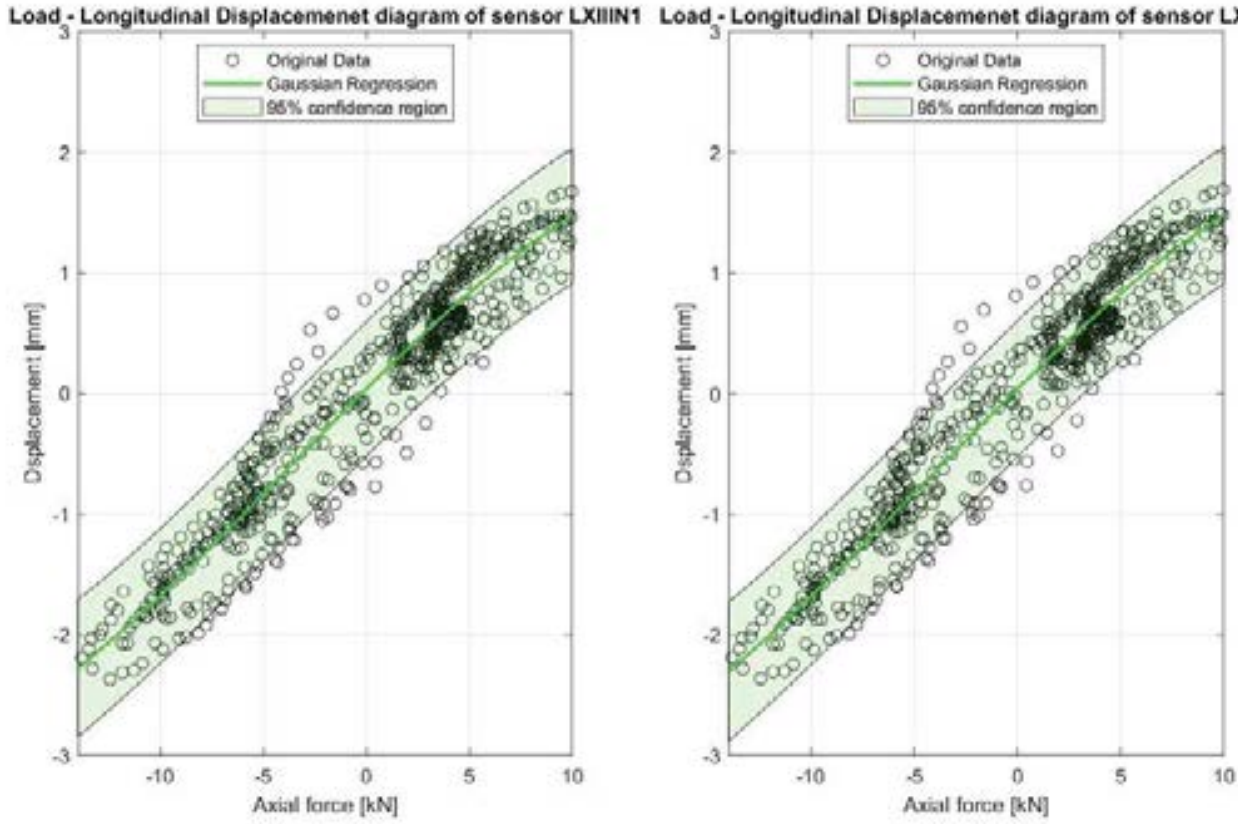

Figure 12: Gaussian Regression of LXIIIN1 and LXIIIN2 after one month of data 


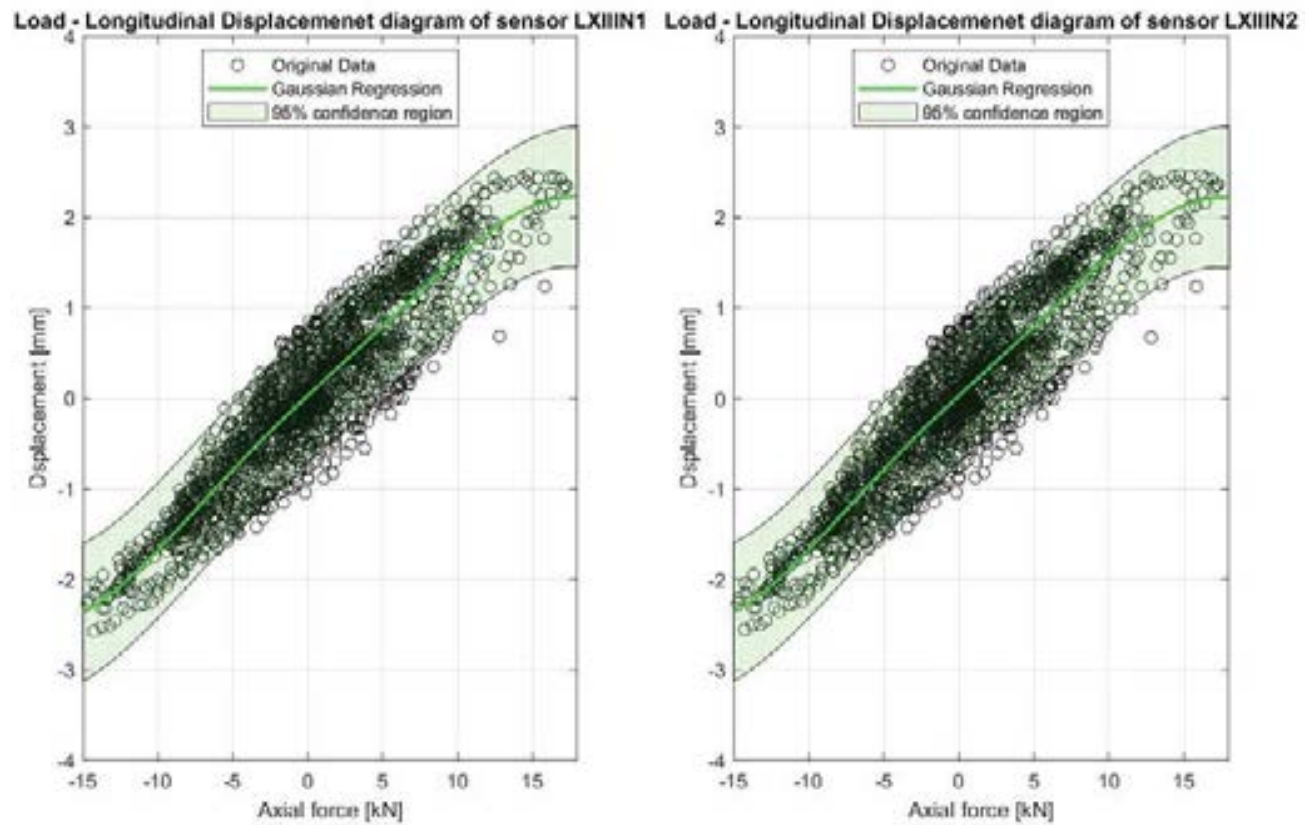

Figure 12: Gaussian Regression of LXIIIN1 and LXIIIN2 after two months of data

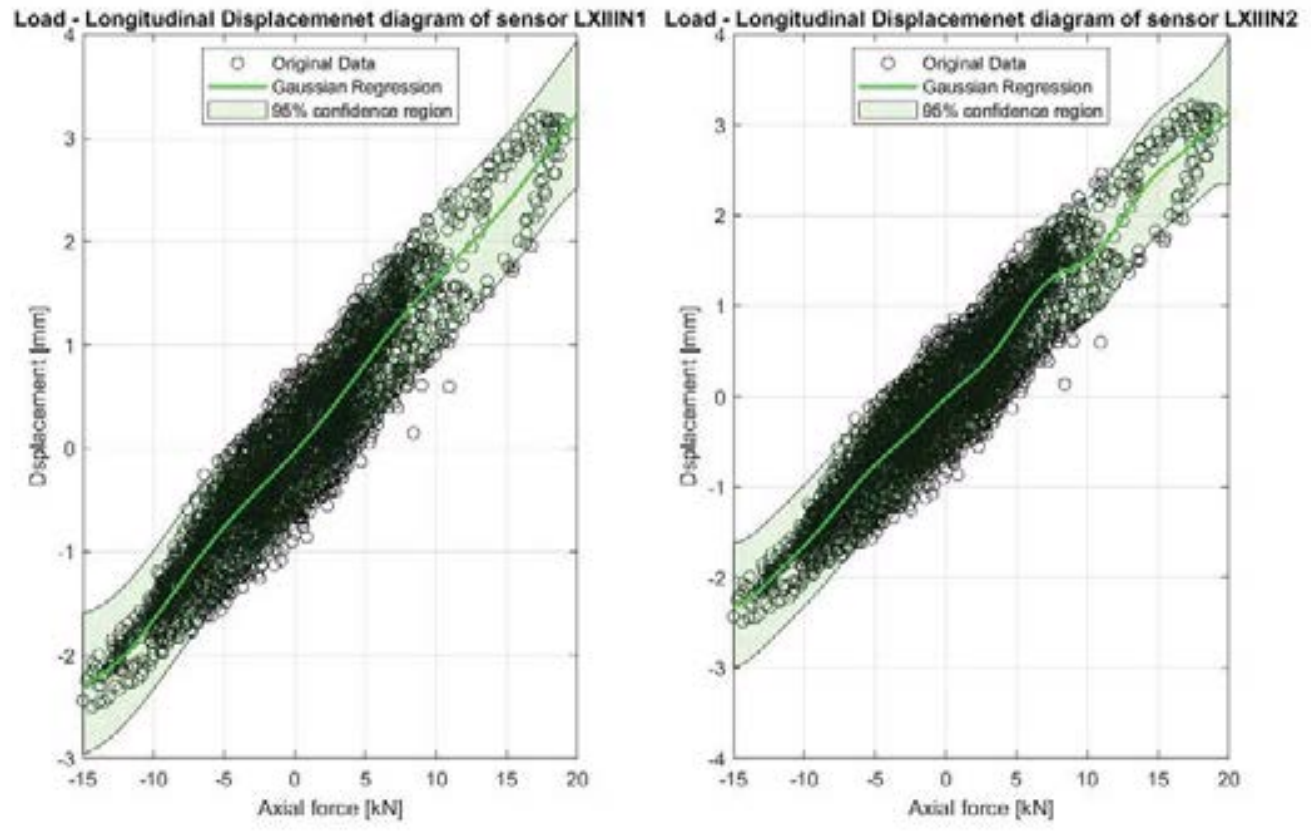

Figure 12: Gaussian Regression of LXIIIN1 and LXIIIN2 after three months of data

\section{ETHICS DECLARATION}

The authors wish to confirm that there are no known conflicts of interest associated with this publication. 


\section{REFERENCES}

[1] L. Sun, Z. Shang, Y. Xia, S. Bhowmick, and S. Nagarajaiah, "Review of Bridge Structural Health Monitoring Aided by Big Data and Artificial Intelligence: From Condition Assessment to Damage Detection," J. Struct. Eng., vol. 146, no. 5, p. 04020073, May 2020, doi: 10.1061/(asce)st.1943-541x.0002535.

[2] C. R. Farrar and K. Worden, "An introduction to structural health monitoring," Philos. Trans. R. Soc. A Math. Phys. Eng. Sci., vol. 365, no. 1851, pp. 303-315, Feb. 2007, doi: 10.1098/rsta.2006.1928.

[3] E. Figueiredo and A. Santos, "Machine learning algorithms for damage detection," in Vibration-Based Techniques for Damage Detection and Localization in Engineering Structures, World Scientific Publishing Co., 2018, pp. 1-40.

[4] Q. Liu, G. Li, R. Jiang, F. Yu, and W. Gai, "An intelligent monitoring method and its experiment for bridge bearing," 2016. Accessed: May 03, 2021. [Online]. Available: https://www.atlantis-press.com/proceedings/iccte-16/25854293.

[5] G.-X. Wang, Y.-L. Ding, Y.-S. Song, L.-Y. Wu, Q. Yue, and G.-H. Mao, "Detection and Location of the Degraded Bearings Based on Monitoring the Longitudinal Expansion Performance of the Main Girder of the Dashengguan Yangtze Bridge," J. Perform. Constr. Facil., vol. 30, no. 4, p. 04015074, Aug. 2016, doi: 10.1061/(asce)cf.1943-5509.0000820.

[6] R. Kromanis, P. Kripakaran, and B. Harvey, "Long-term structural health monitoring of the Cleddau bridge: Evaluation of quasi-static temperature effects on bearing movements," Struct. Infrastruct. Eng., vol. 12, no. 10, pp. 1342-1355, Oct. 2016, doi: 10.1080/15732479.2015.1117113.

[7] D. Garcia-Sanchez, A. Fernandez-Navamuel, D. Z. Sánchez, D. Alvear, and D. Pardo, "Bearing assessment tool for longitudinal bridge performance," J. Civ. Struct. Heal. Monit., vol. 10, no. 5, pp. 1023-1036, Nov. 2020, doi: 10.1007/s13349-020-00432-1.

[8] E. Figueiredo and E. Cross, "Linear approaches to modeling nonlinearities in long-term monitoring of bridges," J. Civ. Struct. Heal. Monit., vol. 3, no. 3, pp. 187-194, Aug. 2013, doi: 10.1007/s13349-013-0038-3. 
[9] C. E. Rasmussen, "Gaussian Processes in machine learning," Lect. Notes Comput. Sci. (including Subser. Lect. Notes Artif. Intell. Lect. Notes Bioinformatics), vol. 3176, pp. 63-71, 2004, doi: 10.1007/978-3-540-28650-9_4.

[10] S. García, S. Ramírez-Gallego, J. Luengo, J. M. Benítez, and F. Herrera, "Big data preprocessing: methods and prospects," Big Data Anal., vol. 1, no. 1, p. 9, Dec. 2016, doi: 10.1186/s41044-016-0014-0.

[11] J. Han, M. Kamber, and J. Pei, "Data Mining. Concepts and Techniques, 3rd Edition (The Morgan Kaufmann Series in Data Management Systems),” 2011.

[12] F. Pozo, I. Arruga, L. E. Mujica, M. Ruiz, and E. Podivilova, "Detection of structural changes through principal component analysis and multivariate statistical inference," Struct. Heal. Monit., vol. 15, no. 2, pp. 127-142, Mar. 2016, doi: 10.1177/1475921715624504.

[13] J. Stanton and J. Taylor, "Friction Coefficients for Stainless Steel/PTFE (teflon) Bearings," 2010 . 